\title{
Validation of a Multi-branched Patient Specific Computational Model for Human Arterial Systems
}

\author{
Yashar Seyed Vahedein, Alexander S Liberson \\ Rochester Institute of Technology \\ James E. Gleason Building, 76 Lomb Memorial Drive, Rochester, NY 14623-5604, United States \\ Yashar.seyed.vahedein@rit.edu; asleme@ rit.edu
}

\begin{abstract}
Cardiovascular waves result from the interaction of the heart with the arterial system and carry significant diagnostic value, often unexploited to its fullest possible degree. The purpose of this work is to create an accurate and robust direct quasi 1D computational model simulating the entire cardiovascular network of an arbitrary topology. This model has a potential to extract information on cardiovascular quantities of interest, for which the direct measurements are not feasible, such as a cardiac output, blood pressure along all arteries of interest, including central aortic pressure, other. Viscoelastic properties of arterial blood vessels of a variable cross section area are formulated based on a Voight linear model. The results are compared with the in-vitro experimental data, replicating the 55 and 37 largest conduit arteries in the human systemic circulation. The presence of viscoelastic properties affects dispersive and dissipative properties of a numerical finite volume scheme, improving results based on a pure linear elastic arterial wall model.
\end{abstract}

Keywords: Hemodynamics, Pulse Wave Velocity, Quasi-1D FSI Modelling, Circulatory System, Direct And Inverse Problems.

\begin{tabular}{|c|c|c|}
\hline \multicolumn{3}{|l|}{ Nomenclature } \\
\hline$A$ & $\left(m^{2}\right)$ & Luminal cross sectional area in a loaded condition \\
\hline$A_{0}$ & $\left(m^{2}\right)$ & Luminal cross sectional area in a load free condition \\
\hline$c_{M K}$ & $(\mathrm{~m} / \mathrm{s})$ & Moens-Korteweg speed of propagation based on a load free cross sectional area \\
\hline $\mathrm{E}$ & $(\mathrm{Pa})$ & Wall linear elastic modulus \\
\hline $\bar{E}$ & $(\mathrm{~Pa})$ & Reduced wall linear elastic modulus \\
\hline \multicolumn{3}{|l|}{$=E /\left(1-v^{2}\right)$} \\
\hline $\mathrm{p}$ & $(\mathrm{Pa})$ & Blood pressure \\
\hline$r_{0}$ & $(\mathrm{~m})$ & Radius of a luminal cross sectional area in a load free condition \\
\hline $\mathrm{u}$ & $(\mathrm{m} / \mathrm{s})$ & Axial velocity averaged across the section \\
\hline$\Gamma$ & $(\mathrm{Pa} * \mathrm{~s})$ & Viscosity constant of a visco-elastic Kelvin-Voight model \\
\hline$\mu$ & $(\mathrm{Pa} * \mathrm{~s})$ & Blood dynamic viscosity \\
\hline$v$ & & Poisson coefficient \\
\hline$\eta$ & & Ratio of a normal displacement to $r_{0}$ \\
\hline$\Delta \mathrm{t}$ & (s) & Time step of a numerical approximation \\
\hline$\Delta x$ & (m) & Mesh spacing of a numerical approximation \\
\hline
\end{tabular}

\section{Introduction}

In the last two decades cardiovascular mathematics, mechanics and numerical modeling have experienced a dramatic advancement. This trend is motivated by the recognized capability of a computational modeling to expand our understanding of physiological and pathological processes, to predict the consequences of surgical intervention, and to assist in surgical planning relating to individual patients. It complements the information obtained by medical imaging or other non-invasive measurements, allowing eventually to predict cardiovascular markers in the areas for which the direct assessment is not feasible. For the detailed literature review on different methods of a cardiovascular modelling and applications the reader is referred to [1-2]. The large scale of a pulse propagating waveform compared to the cross sectional diameter of the vessel significantly reduces the number of parameters required to simulate the fully 3D problems 
(long-wave approximation). A brief review on application of cardiovascular mathematics to solutions of inverse problem can be found in [3].

The current work is an extension of the authors' investigations in areas of direct and inverse problems [4-6]. We present here a finite volume based nonlinear quasi-1D algorithm, with an account for viscoelasticity in tapered vessels of a well-defined experimental model [7].

\section{Methods}

\subsection{Mathematical Model}

Consider the conservation of mass and momentum averaged over cross section to form a 1D system of equations; This is applied to the Newtonian fluid with a constant axisymmetric velocity profile in a visco-elastic tube and a variable cross section area (dot above indicates time derivative, prime - derivative by the axial coordinate)

$$
\dot{Q}+[F(Q)]^{\prime}=f
$$

where

$$
Q=\left[\begin{array}{l}
A \\
u
\end{array}\right] ; \quad F=\left[\begin{array}{c}
A u \\
\frac{u^{2}}{2}+\frac{p}{\rho}
\end{array}\right] ; \quad f=\left[\begin{array}{c}
0 \\
\frac{k u}{A_{0}}
\end{array}\right] ; \quad k=-\frac{22 \mu \pi}{\rho}
$$

The Voight type constitutive equation for the circumferential stress component reads

$$
\sigma=\bar{E} \eta+\Gamma \dot{\eta}
$$

Combining (2) with the equilibrium condition $\sigma=p r_{0} / h$, arrive at the expression for the visco-elastic alternative of a tube law

$$
p=\frac{h}{r_{0}}(\bar{E} \eta+\Gamma \dot{\eta})=2 \rho c_{m k}^{2}(\eta+\beta \dot{\eta}) ; \quad c_{m k}^{2}=\frac{\bar{E} h}{2 \rho r_{0}} ; \quad \beta=\frac{\Gamma}{\bar{E}}
$$

In terms of cross section areas $\left(A=A_{0}(1+\eta)^{2}, \quad \eta=\sqrt{\frac{A}{A_{0}}}-1\right)$, the tube law is presented as the superposition of a an elastic $p_{e}$ and viscous $p_{v}$ components

$$
p=p_{e}-p_{v} ; \quad p_{e}=2 \rho c_{m k}^{2}\left(\sqrt{\frac{A}{A_{0}}}-1\right) ; \quad p_{v}=\frac{\beta \rho c_{m k}^{2}}{2 \sqrt{A A_{0}}}(u A)^{\prime}
$$

In the following, the alternative form of conservation laws (1) will be used. In this form, the flux vector is presented as a function of a pure elastic component, whereas the viscous response is modelled by the source term

$$
F=\left[\begin{array}{c}
A u \\
\frac{u^{2}}{2}+\frac{p_{e}}{\rho}
\end{array}\right] ; \quad f=\left[\frac{k u}{A_{0}}+\frac{p_{v}^{\prime}}{\rho}\right]
$$

The problem given in (1), (5) is solved by the classical Lax-Wendroff scheme of the second order of accuracy in time and space. The derivation here slightly differs from the traditional approach, due to the employment of a Voight's viscouselastic model in a form operating with a space derivative, which affects the numerical differentiation stencil.

From (1) it follows for the $\mathrm{n}^{\text {th }}$ node of a stencil 


$$
\begin{gathered}
\dot{Q}_{n}=\mathrm{f}_{n}-[F(Q)]^{\prime} \\
\ddot{Q}_{n}=\mathrm{f}_{n, n-1} \dot{Q}_{n-1}+\mathrm{f}_{n, n} \dot{Q}_{n}+\mathrm{f}_{n, n+1} \dot{Q}_{n+1}+\left[F_{Q} \dot{Q}\right]_{n}{ }^{\prime}
\end{gathered}
$$

where we have denoted $F_{Q}=\frac{\partial F}{\partial Q} ; \quad \mathrm{f}_{n, k}=\frac{\partial f_{n}}{\partial Q_{k}}, k=n-1, n, n+1$. We now consider the Taylor series expansion truncated at the second order to yield solution at the following time level

$$
Q^{n}=Q_{n}+\tau \dot{Q}_{n}\left(1+\frac{\tau}{2} \mathrm{f}_{n, n}\right)+\frac{\tau^{2}}{2}\left(\mathrm{f}_{n, n-1} \dot{Q}_{n-1}+\mathrm{f}_{n, n+1} \dot{Q}_{n+1}+\left[F_{Q} \dot{Q}\right]_{n}{ }^{\prime}\right)
$$

where the superscript and subscript show the following and the current time levels, relating to the specified node number. The first order time derivatives in (7) are replaced with the space coordinate differentiation according to the first equation (6). The stability criteria for the Lax-Wendroff scheme is

$$
C F L=\frac{\tau\left(u+c_{M K}\right)_{\max }}{\Delta x} \leq 1
$$

All simulation runs are assuming tapered cross sections for the arteries, and a $2666 \mathrm{kPa}$ external pressure. The solution is for 10 cycles, and until a periodic solution is achieved.

\subsection{Boundary Conditions}

Whereas specification of initial conditions does not affect the final solution of a pulsatile flow, correct setting of the boundary conditions should comply with the characteristic properties of a relating mathematical model. To complete the problem consider the Jacobian matrix $F_{Q}$

$$
F_{Q}=\frac{\partial F}{\partial Q}=\left[\begin{array}{cc}
u & A \\
\frac{\rho c_{M K}^{2}}{\sqrt{A A_{0}}} & u
\end{array}\right]
$$

It may be shown that the forward and backward traveling characteristic (Riemann) variables are determined as the following

$$
Y=u+4 c_{M K}\left(\frac{A}{A_{0}}\right)^{1 / 4} ; \quad Z=u-4 c_{M K}\left(\frac{A}{A_{0}}\right)^{1 / 4}
$$

The local speed wave of each characteristic variable is $\lambda_{Y}=u+c_{M K}\left(\frac{A}{A_{0}}\right)^{1 / 4}, \quad \lambda_{Z}=u-c_{M K}\left(\frac{A}{A_{0}}\right)^{1 / 4}$. Since the local Moens-Korteweg speed is significantly larger than the convective velocity, the flow is deeply subcritical. In case of large arteries, in which the convective phenomena is dominant, the specified inlet and exit boundary conditions are complemented by the backward and forward propagating invariants respectfully, preserving their constant values on relating characteristic lines.

\subsubsection{Inflow Boundary Conditions}

With accounts for the flow at the inlet of ascending aorta, we enforce a time dependent flow rate $Q_{0}(t)$. As a result the following system of equations is solved at every time step with respect to the inlet velocity $u_{\text {in }}$ and inlet cross section area $A_{\text {in }}$, 


$$
\begin{gathered}
u_{\text {in }} A_{\text {in }}=Q_{0}(t) \\
u_{\text {in }}-4\left[c_{M K}\left(\frac{A}{A_{0}}\right)^{\frac{1}{4}}\right]_{\text {in }}=Z_{1}
\end{gathered}
$$

where $Z_{1}$ is the backward propagating invariant calculated at the first cell $\mathrm{n}=1$.

\subsubsection{Terminal Boundary Conditions}

Since 1-D model assumptions are not valid for the small arteries, arterioles and capillaries, the terminal conditions are usually specified for the truncated model, construed by a reasonable number of arterial tree generations. Commonly used a three element Windkessel model [ 1,2 ] accounts for the cumulative effects of the distal vessels beyond the terminal site, introducing proximal and distal resistances $R_{1}, R_{2}$, and a compliance of the vascular bed $C_{T}$. This model relates time dependent pressure and flow at the truncated section, being expressed in differential form, as shown by the $1^{\text {st }}$ equation of (12). The terminal condition is complemented by the forward propagating invariant $Y_{N}$, calculated at the last cell, $n=N$

$$
\begin{gathered}
(\dot{u A})_{T}=\left[\frac{\dot{p}_{e}}{R_{1}}+\frac{p_{e}}{R_{1} R_{2} C_{T}}-\left(1+\frac{R_{1}}{R_{2}}\right) \frac{u A}{R_{1} C_{T}}\right]_{T} \\
u_{T}+4\left[c_{M K}\left(\frac{A}{A_{0}}\right)^{\frac{1}{4}}\right]_{T}=Y_{N}
\end{gathered}
$$

where subscript " $T$ " indicates terminal conditions.

\subsubsection{Arterial Bifurcation}

Splitting flow junctions are the typical alignments in arterial systems. The parent vessel designated as the vessel 1 (Fig. 1) connects by its outlet to the inlets of the daughter tubes, noted as vessels 2 and 3. Let $U_{1}, P_{1} A_{1}$ indicate velocity, pressure and cross section area of a parent vessel at the junction interface, $U_{2}, P_{2} A_{2}, U_{3}, P_{3} A_{3}$ - the corresponding properties of daughters' vessels at the interface (Fig.1). The following assumptions are made to simplify the junction matching procedure: (1) energy losses at the junctions are ignored, (2) pressure change across the junction is neglected, $P_{1}=P_{2}=P_{3}=P$. Since the pressure values algebraically relate to the cross section areas (4), the only four independent quantities $U_{1}, U_{2}, U_{3}, P$ need to be specified. To close the problem the conservation of mass and extrapolation of forward and backward propagating invariants present

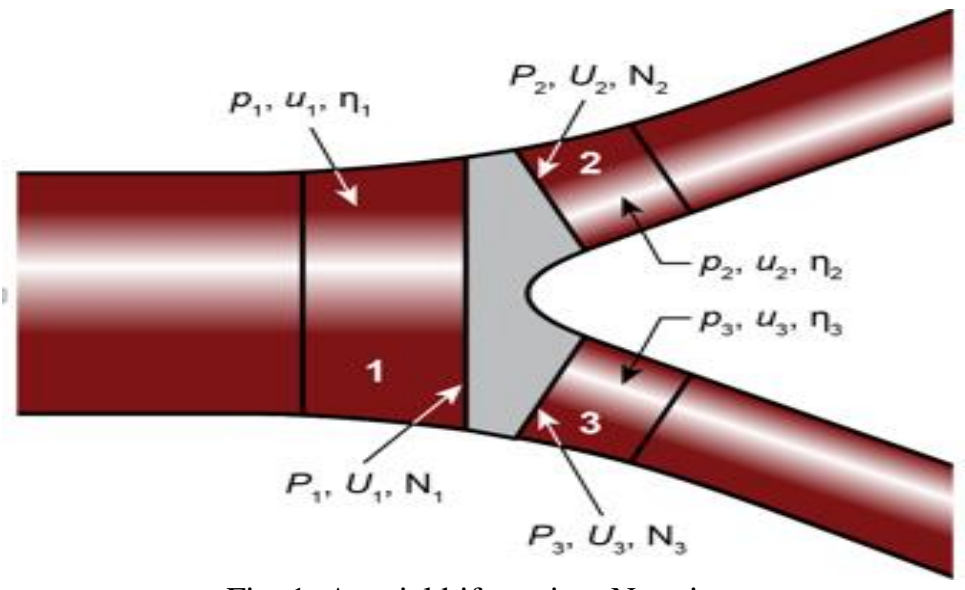

Fig. 1: Arterial bifurcation. Notation. 


$$
\begin{gathered}
U_{1} A_{1}=U_{2} A_{2}+U_{3} A_{3} \\
U_{1}+4\left[c_{M K}\left(\frac{A}{A_{0}}\right)^{\frac{1}{4}}\right]_{1}=Y_{1} \\
U_{2}-4\left[c_{M K}\left(\frac{A}{A_{0}}\right)^{\frac{1}{4}}\right]_{2}=Z_{2} \\
U_{3}-4\left[c_{M K}\left(\frac{A}{A_{0}}\right)^{\frac{1}{4}}\right]_{3}=Z_{3}
\end{gathered}
$$

where $Y_{1}, Z_{2}, Z_{3}$ are characteristic variables calculated at the last cell of a parent vessel and the first cell of the daughters vessels (Fig.1).

\section{Applications}

\subsection{Model of the 55 Main Systemic Human Arteries}

The 55 arteries of the in vitro experimental model from [7] are simulated within the nonlinear formulation frame. Each arterial domain is characterized by its length, cross sectional area, Moens-Korteweg (acoustic) speed of propagation, and a linear elastic modulus. Flow is a laminar Newtonian liquid formed by a mixture of $70 \%$ water and $30 \%$ of glycerine. The pulse duplicator system was operating at 60 beats/min, producing a cardiac output of $4.1 \mathrm{l} / \mathrm{min}$. At each terminal branch the simplest no-reflected boundary conditions have been specified. The pressure and velocity waveforms are presented in Figure 2. Convergence for the vascular tree was achieved in a minute, using PC with a dual core $2.4 \mathrm{~Hz}$ i5 Intel processor. To verify results across measured data each truncated vessel should be assigned to the three element Windkessel model.

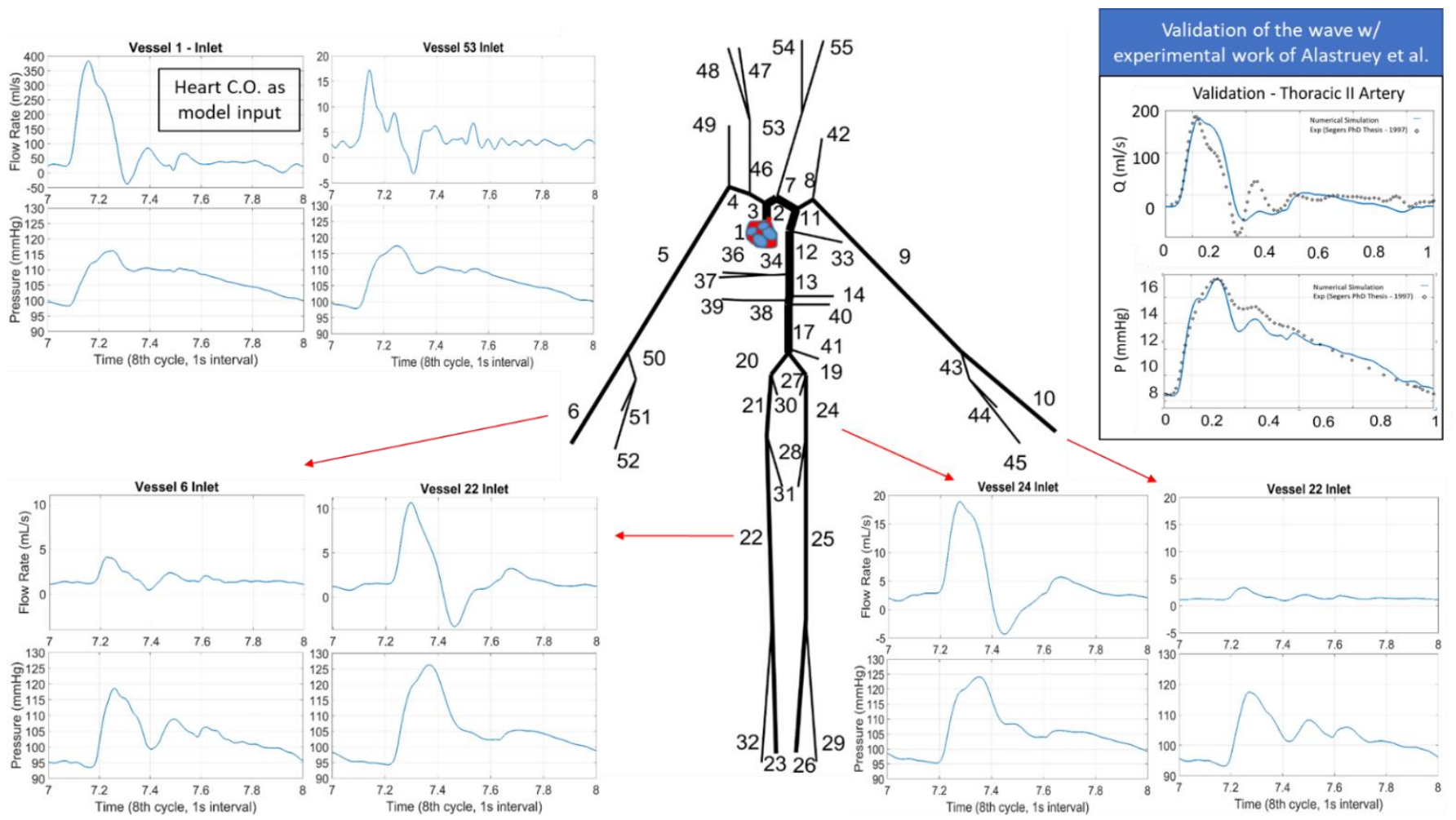

Fig. 2: Pressure and flow rate time histories in the middle points of selected arteries, validation and flow inlet from Segers and Alastruey et al. [8-9]). 
Fig. 2 compares pressure and flow rate time histories at different locations along the ascending, thoracic and abdominal aorta obtained with the experimental and numerical models once a periodic solution has been achieved. The values of the mean pressure along the aorta are well predicted by the 1-D method, with relative error below $2 \%$. The simulation approximates well the slope of the diastolic decay, and waveforms of pressure and flow rate distributions. Time history of the flow rate approximates well in proximity of the peak flow, whereas low level of flow deviates from experiment at some time instants by $20 \%$. This study increases the confidence in the 1D code and its ability to model clinically relevant problems, such as applications presented in this proposal.

\subsection{Addition of Vessel Wall Viscoelasticity}

A model of the 37 main arteries, as presented both numerically and experimentally by Matthys et al. [10], is simulated and solved with our in-house algorithm. Matthys et al., hypothesized that viscoelastic properties of blood vessels may affect the predicted blood pressure and flow rate as a function of time, and improve the accuracy. Here we set the viscoelastic properties using the measurements presented in Alastruey et al. [11], in which they experimentally measure the wall viscoelastic parameter $(\Gamma)$. Note that the $\Gamma$ presented in section 2 is slightly different than [11]. In Fig. 3, two sets of pressure waveform results along the aorta are compared; $\mathrm{a}, \mathrm{b}$ and $\mathrm{c}$ are from our in-house quasi 1D nonlinear model for a pure elastic case, and $\mathrm{d}, \mathrm{e}$, and $\mathrm{f}$ represent the corresponding results from a viscoelastic counterpart. As hypothesized by Matthys et al., we can see that the results of a viscoelastic case are smoother and thus closer to the experimentally measured pressures. Our algorithm for the viscoelasticity is enforcing the viscoelastic properties as source terms of the conservation equations. This is a simplified alternative to the formulation presented in Alastruey et al. [11]; however, the smoothing effect in the flux-based formulation, presented in [11], has a more rigorous effect on dampening the extra oscillations in the results.
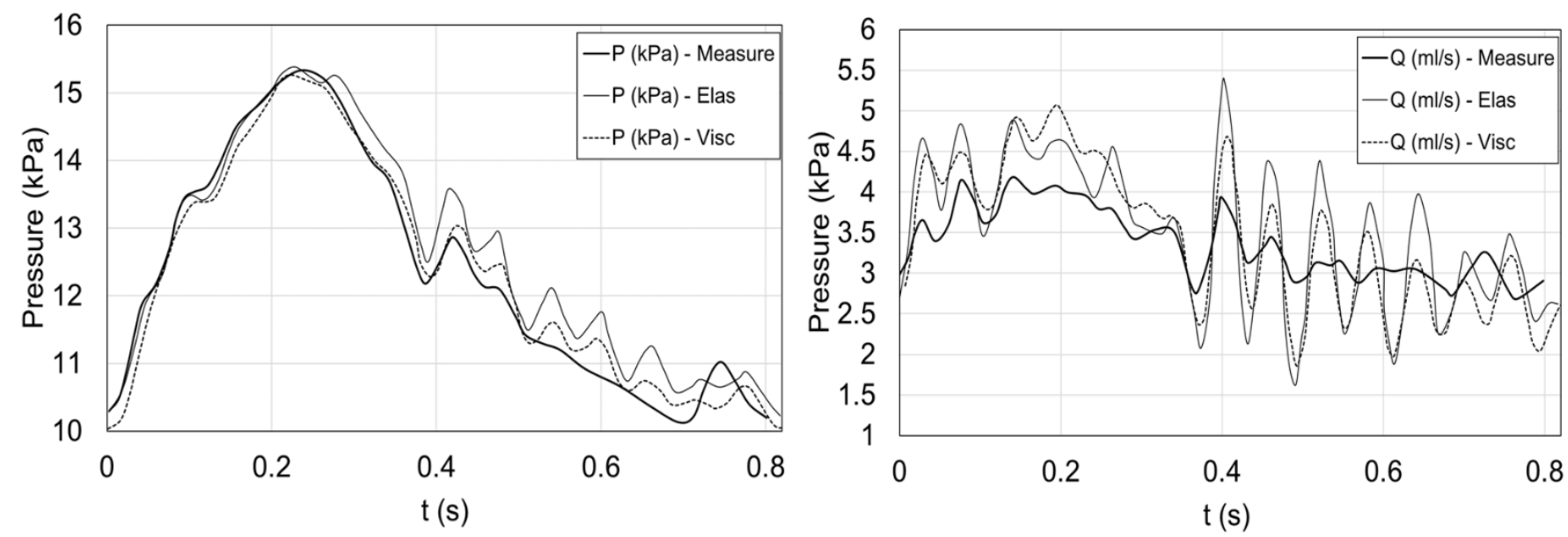

Fig. 3: Pressure and flow rate time histories in the "Left Renal" artery (\#24), for viscoelastic, elastic and experimental models. Elastic solution results and experimental measurement reproduced from [11].

With the current Lax-Wendroff formulation the difference between the elastic and source-term, based viscoelastic prediction is less than $0.5 \%$. However, we show a significant improvement compared to the elastic model demonstrated in Matthys et al. [10] with both elastic and viscoelastic models.

\section{Conclusion}

We have developed a tool to accurately estimate the arterial wave mechanics in the systemic circulation based on a quasi-1D formulation. Long wave approximation significantly reduces the number of parameters required to simulate the fully 3D problem. Numerical results produce an excellent match with an in vitro measurement in a 55 vessels arterial tree. Additionally, the effect of adding a source term based viscoelastic formulation is demonstrated. We show that the viscoelasticity provides smoother results compared to the elastic counterpart, resulting to a closer match with the experimental measurements. This algorithm is applicable in diastolic blood pressure prediction and inverse prediction of the cardiovascular biomarkers. 


\section{References}

[1] L. Formaggia, A. Quarteroni, A. Veneziani, eds. "Cardiovascular mathematics," Springer, 2009.

[2] F. N. Vosse, N. Stergiopulos, "Pulse wave propagation in the arterial tree," Апnи Rev. Fluid Mech, vol. 43, pp. 467499, 2011.

[3] A. Veneziani, C. Vergara, "Inverse problems in cardiovascular mathematics," Int. J. Numer. Meth. Biomed. Engng., vol. 29, pp. 723-725, 2013.

[4] A. S. Liberson, J. S. Lillie, D. A. Borkholder, "A Physics based approach to the pulse wave velocity prediction in compliant arterial segments," Journal of Biomechanics, 2016.

[5] A. S. Liberson Y. S. Vahedein, D. A. Borkholder, "Application of variational principle to form reduced fluidstructure interaction models in bifurcated networks," Journal of Fluid Flow, Heat and Mass Transfer., vol. 4, 2017.

[6] Y. S. Vahedein, A. S. Liberson, "Reduced modelling framework of circulatory system revisited," Proc. FFHMT'17, no. 174.

[7] J. Alastruey, A. W. Khir, K. S. Matthys, P. Segers, S.W. Sherwin, P. R. Verdonck, K. H. Parker, J. Peiro, "Pulse wave propagation in a model human arterial network. Assesment of $1 \mathrm{D}$ visco-elastic simulations against in vitro measurements," Journal of Biomechanics, vol. 44, pp. 2250-2258, 2011.

[8] J. A. Alastruey, "Numerical Modelling of Pulse Wave Propagation in the Cardiovascular System: Development, Validation and Clinical Applications," Ph.D. Thesis, University of London, 2006.

[9] P. Segers, "Biomedical modelling of the arterial system for the non-invasive assessment of arterial compliance (Biomechanische modellering van het arterieel systeem voor de niet-invasieve bepaling van de arterimboxële compliantie)," Ph.D. Thesis, Ghent University, 1997.

[10] K. S. Matthys et al., "Pulse wave propagation in a model human arterial network: Assessment of 1-D numerical simulations against in vitro measurements," J. Biomech., vol. 40, no. 15, pp. 3476-3486, 2007.

[11] J. Alastruey, et al, "Pulse Wave Propagation in a Model Human Arterial Network: Assessment of 1-D Visco-Elastic Simulations against in VitroMeasurements," Journal of Biomechanics, vol. 44, no. 12, pp. 2250-2258, 2011. PMC. Web. 13 Feb. 2018. 